\title{
Article
}

\section{The effect of time-varying delay damping on the stability of porous elastic system}

\section{Soh Edwin Mukiawa}

Department of Mathematics, University of Hafr Al Batin, Hafr Al Batin 39524, Saudi Arabia; mukiawa@uhb.edu.sa Academic Editor: Gayrat Urazboev Received: 18 January 2021; Accepted: 8 March 2021; Published: 28 March 2021.

Abstract: In the present work, we study the effect of time varying delay damping on the stability of a one-dimensional porous-viscoelastic system. We also illustrate our findings with some examples. The present work improve and generalize existing results in the literature.

Keywords: Optimal decay; Porous system; Delay; Viscoelasticity; Convexity.

MSC: 35B35; 35D35; 35B40; 93D20.

\section{Introduction}

roblems in porous media are essential in the field of petroleum engineering, soil mechanics, power technology, biology, material science, etc. Thus, this has attracted the attention of scientists and mathematicians in particular, see for instance the results in [1-8] and the references cited therein for related theory of porous-elastic materials. The basic equations describing the motion of a classical porous system are given by

$$
\begin{cases}\rho \varphi_{t t}-S_{x}=0, & \text { in }(0, L) \times \mathbb{R}_{+}, \\ J \psi_{t t}-G_{x}-Q=0, & \text { in }(0, L) \times \mathbb{R}_{+},\end{cases}
$$

where $\varphi=\varphi(x, t)$ and $\psi=\psi(x, t)$ are the displacements of solid elastic material and the volume fraction, respectively. The physical parameters $\rho$ and $J$ are respectively, mass density and product of the equilibrated inertia by the mass density. The constitutive laws $S, G$ and $Q$ are: stress tensor, equilibrated stress vector and equilibrated body force, respectively. Time delays occur in systems modeling different types of phenomena in areas such as: biosciences, medicine, physics, chemical and structural engineering. These phenomena depend naturally on the present state and past history of the system. It is a well known fact that the presence of a delay term in a system, which is a priori a stable system, might cause an instability in the system, see for instance, the result of Nicaise and Pignotti [9]. In past decades, a great number of researchers have investigated the effect of delay on the stability of various systems or wave equations (with or without memory), see for example [10-15] and references therein. Back to system (1), we should mention that, there are very few results in literature that studied the effect of delay on this system. With memory and time varying delay dampings, the constitutive laws in (1) are given by

$$
\left\{\begin{array}{l}
S=k \varphi_{x}+b \psi, \\
G=\delta \psi_{x}-\int_{0}^{t} g(t-s) \psi_{x}(., s) d s, \\
Q=-b \varphi_{x}-a \psi-\mu_{1} \psi_{t}-\mu_{2} \psi_{t}(., t-\tau(t)),
\end{array}\right.
$$

where the constitutive physical parameters, $k, b, \delta, a$ satisfies

$$
k>0, \delta>0, a>0, b^{2}<k a,
$$

$\mu_{1}, \mu_{2}$ are real constants, $\tau(t)>0$ is the time-dependent delay and $g$ is a given function to be specified later. For simplicity, we set $L=1$, then substituting (2) into (1), we arrive at the following porous-viscoelastic system with varying time dependent delay; 


$$
\begin{cases}\rho \varphi_{t t}-k \varphi_{x x}-b \psi_{x}=0, & \text { in }(0,1) \times \mathbb{R}_{+}, \\ J \psi_{t t}-\delta \psi_{x x}+b \varphi_{x}+a \psi+\int_{0}^{t} g(t-s) \psi_{x x}(x, s) d s+\mu_{1} \psi_{t}+\mu_{2} \psi_{t}(., t-\tau(t))=0, & \text { in }(0,1) \times \mathbb{R}_{+}, \\ \varphi_{x}(0, t)=\varphi_{x}(1, t)=\psi(0, t)=\psi(1, t)=0, & t \in \mathbb{R}_{+}, \\ \varphi(x, 0)=\varphi_{0}(x), \varphi_{t}(x, 0)=\varphi_{1}(x), \psi(x, 0)=\psi_{0}(x), \psi_{t}(x, 0)=\psi_{1}(x), x \in(0,1), & \\ \psi_{t}(x, t)=f_{0}(x, t), \quad \text { in }(0,1) \times(-\tau(0), 0) . & \end{cases}
$$

When $g=\mu_{1}=\mu_{2}=0$, Quintanilla [16] investigated

$$
\begin{cases}\rho \varphi_{t t}-k \varphi_{x x}-b \psi_{x}=0, & \text { in }(0,1) \times(0,+\infty), \\ J \psi_{t t}-\delta \psi_{x x}+b \varphi_{x}+a \psi-\gamma \psi_{t}=0, & \text { in }(0,1) \times(0,+\infty),\end{cases}
$$

where $\mu_{1}=\gamma>0$ and showed the lack of exponential stability. However, he established a slow non-exponential decay result. Casas and Quintanilla [17] studied

$$
\left\{\begin{array}{lr}
\rho \varphi_{t t}-k \varphi_{x x}-b \psi_{x}+\beta \theta_{x}=0, & \text { in }(0,1) \times(0,+\infty), \\
J \psi_{t t}-\delta \psi_{x x}+b \varphi_{x}+a \psi-m \theta+\gamma \psi_{t}=0, & \text { in }(0,1) \times(0,+\infty), \\
c \theta_{t}-\kappa \theta_{x x}+\beta \varphi_{x t}+m \psi_{x t}=0, & \text { in }(0,1) \times(0,+\infty),
\end{array}\right.
$$

and improved the result in [16] (exponential stability). Soufyane et al., [18] considered (5) with viscoelastic damping on the boundaries and proved a general decay estimate. Recently, Apalara [19] looked at

$$
\begin{cases}\rho \varphi_{t t}-k \varphi_{x x}-b \psi_{x}=0, & \text { in }(0,1) \times(0,+\infty), \\ J \psi_{t t}-\delta \psi_{x x}+b \varphi_{x}+a \psi+\int_{0}^{t} g(t-s) \psi_{x x}(x, s) d s=0, & \text { in }(0,1) \times(0,+\infty),\end{cases}
$$

where the memory $g$ satisfies $g^{\prime}(t) \leq-\xi(t) g(t)$ and established a general decay estimate. Feng and Apalara [20] improved the result in [19] when the relaxation function $g$ satisfies $g^{\prime}(t) \leq-\xi(t) H(g(t))$.

For results in porous systems with delay damping, not much has been done in this direction. we refer the reader to the result of Khochemane et al., [21], where they considered a porous elastic system with weak internal damping and constant delay damping. Precisely, they studied

$$
\begin{cases}\rho \varphi_{t t}-k \varphi_{x x}-b \psi_{x}=0, & \text { in }(0,1) \times(0,+\infty), \\ J \psi_{t t}-\delta \psi_{x x}+b \varphi_{x}+a \psi+\alpha(t) g\left(\psi_{t}\right)+\mu_{1} \psi_{t}+\mu_{2} \psi_{t}(., t-\tau)=0, & \text { in }(0,1) \times(0,+\infty),\end{cases}
$$

and proved a general decay result provided $g$ satisfies

$$
\begin{cases}c_{1}|s| \leq|g(s)| \leq c_{2}|s|, & \text { if }|s| \geq \epsilon \\ s^{2}+g^{2}(s) \leq G^{-1}(s g(s)), & \text { if }|s| \leq \epsilon\end{cases}
$$

Recently, Borges Filho and Santos [22] considered

$$
\begin{cases}\rho \varphi_{t t}-k \varphi_{x x}-b \psi_{x}=0, & \text { in }(0,1) \times(0,+\infty), \\ J \psi_{t t}-\delta \psi_{x x}+b \varphi_{x}+a \psi+\mu_{1} \psi_{t}+\mu_{2} \psi_{t}(., t-\tau(t))=0, & \text { in }(0,1) \times(0,+\infty),\end{cases}
$$

and showed that the system is exponentially stable. More related results can be found in [23-27] and references therein.

The novelty of this work is to study the stability of system (7). In fact, we show that the solution energy has an optimal decay estimate even in the presence of time varying delay term, from which the results in $[21,22]$ are particular cases. To the best of our knowledge, system (7) has not been considered before in the literature. The rest of work is organized as follows: In Section 2, we recall some preliminaries and assumptions on the memory term. In Section 3, we state and prove several lemmas needed for establishing our main results. In Section 4, we establish the uniform stability result and in Section 5, we give some examples in support of our results. 


\section{Problem setting and assumptions}

In this work, we consider the following system:

$$
\begin{cases}\rho \varphi_{t t}-k \varphi_{x x}-b \psi_{x}=0, & \text { in }(0,1) \times \mathbb{R}_{+}, \\ J \psi_{t t}-\delta \psi_{x x}+b \varphi_{x}+a \psi+\int_{0}^{t} g(t-s) \psi_{x x}(x, s) d s+\mu_{1} \psi_{t}+\mu_{2} \psi_{t}(., t-\tau(t))=0, & \text { in }(0,1) \times \mathbb{R}_{+}, \\ \varphi_{x}(0, t)=\varphi_{x}(1, t)=\psi(0, t)=\psi(1, t)=0, & t \in \mathbb{R}_{+}, \\ \varphi(x, 0)=\varphi_{0}(x), \varphi_{t}(x, 0)=\varphi_{1}(x), \psi(x, 0)=\psi_{0}(x), \psi_{t}(x, 0)=\psi_{1}(x), & x \in(0,1), \\ \psi_{t}(x, t)=f_{0}(x, t), \quad \text { in }(0,1) \times(-\tau(0), 0) . & \end{cases}
$$

In addition to (3), we need the following:

\section{Assumptions}

(A1) The relaxation function $g:[0,+\infty) \rightarrow(0,+\infty)$ is a $\mathcal{C}^{1}$ non-increasing function and satisfies

$$
g(0)>0, \quad \delta-\int_{0}^{+\infty} g(s) d s=l>0 .
$$

(A2) There exists a $\mathcal{C}^{1}$-function $M:[0,+\infty) \rightarrow[0,+\infty)$, which is either linear or is a strictly increasing and strictly convex $\mathcal{C}^{2}$ function on $[0, \alpha], \alpha>0, \alpha \leq g(0)$, with $M(0)=M^{\prime}(0)=0$, such that

$$
g^{\prime}(t) \leq-\xi(t) M(g(t)), \quad \forall t \geq 0,
$$

where $\xi$ is a positive non-increasing differentiable function.

(A3) There exist $\tau_{0}, \tau_{1}>0$ such that

$$
0<\tau_{0} \leq \tau(t) \leq \tau_{1}, \forall t>0
$$

(A4)

$$
\tau(t) \in W^{2,+\infty}(0, T) \text { and } \tau^{\prime}(t) \leq d<1, \forall t, T>0 .
$$

(A5) The real constants $\mu_{1}$ and $\mu_{2}$ satisfies $\left|\mu_{2}\right|<\mu_{1} \sqrt{1-d}$.

From $(A 1)$ and $(A 2)$, we can deduce the following:

(I) It follows from $(A 1)$ that $\lim _{t \rightarrow \infty} g(t)=0$. Thus, there exists $t_{0} \geq 0$ large enough, such that

$$
g\left(t_{0}\right)=\alpha \quad \text { and } \quad g(t) \leq \alpha, \quad \forall t \geq t_{0}
$$

(II) Since $g$ and $\xi$ are positive, non-increasing and continuous functions, in addition to $M$ being a positive continuous function, it follows that, for all $t \in\left[0, t_{0}\right]$,

$$
\left.\begin{array}{l}
0<g\left(t_{0}\right) \leq g(t) \leq g(0) \\
0<\xi\left(t_{0}\right) \leq \xi(t) \leq \xi(0)
\end{array}\right\} \Rightarrow \beta_{1} \leq \xi(t) M(g(t)) \leq \beta_{2}
$$

for some positive constants $\beta_{1}$ and $\beta_{2}$. Hence,

$$
g^{\prime}(t) \leq-\xi(t) M(g(t)) \leq-\frac{\beta_{1}}{g(0)} g(0) \leq-\frac{\beta_{1}}{g(0)} g(t), \forall t \in\left[0, t_{0}\right]
$$

(III) $M$ has an extension $\bar{M}$, which is a strictly increasing and strictly convex $\mathcal{C}^{2}$ function on $(0, \infty)$. As an example, given that $M(\alpha)=a_{1}, M^{\prime}(\alpha)=a_{2}$ and $M^{\prime \prime}(\alpha)=a_{3}$, then we can define $\bar{M}$ by

$$
\bar{M}(t)=\frac{a_{3}}{2} t^{2}+\left(a_{2}-a_{3} \alpha\right) t+\left(a_{1}+\frac{a_{3}}{2} \alpha^{2}-a_{2} \alpha\right), \forall t>\alpha
$$

From now on, $C$ denotes a positive constant that may change within lines or from line to line. We denote by $\|\cdot\|_{2}$ the usual norm in $L^{2}(0,1)$ and define the following spaces:

$$
L_{*}^{2}(0,1)=\left\{w \in L^{2}(0,1): \int_{0}^{1} w(x) d x=0\right\}, H_{*}^{1}(0,1)=H^{1}(0,1) \cap L_{*}^{2}(0,1),
$$


and

$$
H_{*}^{2}(0,1)=H^{2}(0,1) \cap H_{*}^{1}(0,1) .
$$

Let $W=\left(\varphi, \varphi_{t}, \psi, \psi_{t}\right), W_{0}=\left(\varphi_{0}, \varphi_{1}, \psi_{0}, \psi_{1}\right)$ and set $\quad \mathcal{H}=H_{*}^{1}(0,1) \times L_{*}^{2}(0,1) \times H_{0}^{1}(0,1) \times L^{2}(0,1), \quad \mathcal{H}_{1}=$ $H_{*}^{2}(0,1) \times H_{*}^{1}(0,1) \times H^{2}(0,1) \cap H_{0}^{1}(0,1) \times H_{0}^{1}(0,1)$. We have the following well-possedness result, which is obtained by using the Classical Faedo-Galerkin method.

Theorem 1. Suppose assumptions $(A 1)-(A 5)$ hold. Let $W_{0} \in \mathcal{H}$ and $f_{0} \in H^{1}((0,1) \times(-\tau(0), 0))$, then (7) possesses a unique weak solution $W \in \mathcal{C}([0,+\infty), \mathcal{H})$. Moreover, if $W_{0} \in \mathcal{H}_{1}$ and $f_{0} \in H^{2}((0,1) \times(-\tau(0), 0))$, then the solution is more regular in the class $W \in \mathcal{C}\left([0,+\infty), \mathcal{H}_{1}\right) \cap \mathcal{C}^{1}([0,+\infty), \mathcal{H})$.

We recall the following useful lemmas that will be applied repeatedly throughout this article.

Lemma 1. Let $w \in L_{\text {loc }}^{2}\left([0,+\infty), L^{2}(0,1)\right)$, we have

$$
\int_{0}^{1}\left(\int_{0}^{t} g(t-s)(w(t)-w(s)) d s\right)^{2} d x \leq(1-l)(g \circ w)(t)
$$

and

$$
\int_{0}^{1}\left(\int_{0}^{x} w(y, t) d y\right)^{2} d x \leq\|w(t)\|_{2}^{2}
$$

where $(g \circ w)(t)=\int_{0}^{t} g(t-s)\|w(t)-w(s)\|_{2}^{2} d s$.

Lemma 2. Let $w \in H_{0}^{1}(0,1)$, then

$$
\int_{0}^{1}\left(\int_{0}^{t} g(t-s)(w(t)-w(s)) d s\right)^{2} d x \leq C_{p}(1-l)(g \circ w)(t),
$$

where $C_{p}>0$ is the Poincaré's constant and $(g \circ w)(t)=\int_{0}^{t} g(t-s)\|w(t)-w(s)\|_{2}^{2} d s$.

Lemma 3. Let $(\varphi, \psi)$ be the solution of (7). Then, for any $0<\alpha<1$, we have

$$
\int_{0}^{1}\left(\int_{0}^{t} g(t-s)\left(\psi_{x}(s)-\psi_{x}(t)\right) d s\right)^{2} d x \leq A_{\alpha}\left(h \circ \psi_{x}\right)(t)
$$

where $h(t)=\alpha g(t)-g^{\prime}(t)$ and $A_{\alpha}=\int_{0}^{+\infty} \frac{g^{2}(s)}{\alpha g(s)-g^{\prime}(s)} d s$.

Proof. Cauchy-Schwarz inequality gives

$$
\begin{aligned}
\int_{0}^{1}\left(\int_{0}^{t} g(t-s)\left(\psi_{x}(s)-\psi_{x}(t)\right) d s\right)^{2} d x & =\int_{0}^{1}\left(\int_{0}^{t} \frac{g(t-s)}{\sqrt{h(t-s)}} \sqrt{h(t-s)}\left(\psi_{x}(s)-\psi_{x}(t)\right) d s\right)^{2} d x \\
& \leq\left(\int_{0}^{+\infty} \frac{g^{2}(s)}{h(s)} d s\right) \int_{0}^{1} \int_{0}^{t} h(t-s)\left(\psi_{x}(s)-\psi_{x}(t)\right)^{2} d s d x \\
& =A_{\alpha}\left(h \circ \psi_{x}\right)(t) .
\end{aligned}
$$

Lemma 4 (Jensen's inequality). Given that $G$ is a convex function on $[a, b], f: \Omega \rightarrow[m, n]$ and $h$ are integrable functions on $\Omega, q(x) \geq 0$, and $\int_{\Omega} q(x) d x=\varrho>0$, then

$$
G\left[\frac{1}{\varrho} \int_{\Omega} f(x) q(x) d x\right] \leq \frac{1}{\varrho} \int_{\Omega} G[f(x)] q(x) d x .
$$

\section{Strategic lemmas}

For convenience, we will denote the norm $\|\cdot\|_{L^{2}(0,1)}$ and the inner product $\langle. . .\rangle_{L^{2}(0,1)}$ of the Lebesgue space $L^{2}(0,1)$ by $\|\cdot\|$ and $\langle.,$.$\rangle respectively. The constants c>0$ and $C>0$ are generic constants which may 
change in value from one line to the other or within the same line. We define the energy functional of problem (7) as

$$
\begin{aligned}
E(t)= & \frac{\rho}{2}\left\|\varphi_{t}\right\|^{2}+\frac{k}{2}\left\|\varphi_{x}\right\|^{2}+\frac{J}{2}\left\|\psi_{t}\right\|^{2}+\frac{a}{2}\|\psi\|^{2}+\frac{1}{2}\left(\delta-\int_{0}^{t} g(s) d s\right)\left\|\psi_{x}\right\|^{2} \\
& +\frac{1}{2}\left(g \circ \psi_{x}\right)(t)+b\left\langle\varphi_{x}, \psi\right\rangle+\frac{\zeta}{2} \int_{t-\tau(t)}^{t} e^{-\lambda(t-s)}\left\|\psi_{t}(s)\right\|^{2} d s,
\end{aligned}
$$

where $\zeta>0$ is a constant to be specified later, see [28] and $\lambda>0$ satisfies

$$
0<\lambda<\frac{2}{\tau_{1}} \log _{e}\left(\frac{\mu_{1}}{\left|\mu_{2}\right|} \sqrt{1-d}\right)
$$

and $\left(g \circ \psi_{x}\right)(t)=\int_{0}^{t} g(t-s)\left\|\psi_{x}(t)-\psi_{x}(s)\right\|^{2} d s$.

Lemma 5. Assume the conditions (A1) - (A5) hold. Then, the energy functional (20) satisfies

$$
\begin{aligned}
E^{\prime}(t) \leq & \frac{1}{2}\left(g^{\prime} \circ \psi_{x}\right)-\frac{1}{2} g(t)\left\|\psi_{x}\right\|^{2}-\left(\frac{\mu_{1}}{2}-\frac{\zeta}{2}\right)\left\|\psi_{t}\right\|^{2} \\
& -\left[\frac{\zeta}{2} e^{-\lambda \tau_{1}}(1-d)-\frac{\mu_{2}^{2}}{2 \mu_{1}}\right]\left\|\psi_{t}(t-\tau(t))\right\|^{2} \\
& -\frac{\lambda \zeta}{2} \int_{t-\tau(t)}^{t} e^{-\lambda(t-s)}\left\|\psi_{t}(s)\right\|^{2} d s \leq 0, \quad \forall t \geq 0 .
\end{aligned}
$$

Proof. Differentiation of (20) gives

$$
\begin{aligned}
E^{\prime}(t)= & \rho\left\langle\varphi_{t}, \varphi_{t t}\right\rangle+k\left\langle\varphi_{x}, \varphi_{x t}\right\rangle+J\left\langle\psi_{t}, \psi_{t t}\right\rangle+a\left\langle\psi, \psi_{t}\right\rangle+b \frac{d}{d t}\left\langle\varphi_{x}, \psi\right\rangle \\
& +\frac{1}{2} \frac{d}{d t}\left[\left(1-\int_{0}^{t} g(s) d s\right)\left\|\psi_{x}(t)\right\|^{2}\right]+\frac{1}{2} \frac{d}{d t}\left(g \circ \psi_{x}\right)(t) \\
& +\frac{\zeta}{2}\left\|\psi_{t}\right\|^{2}-\frac{\zeta}{2} e^{-\lambda \tau(t)}\left(1-\tau^{\prime}(t)\right)\left\|\psi_{t}(t-\tau(t))\right\|^{2} \\
& -\frac{\lambda \zeta}{2} \int_{t-\tau(t)}^{t} e^{-\lambda(t-s)}\left\|\psi_{t}(s)\right\|^{2} d s .
\end{aligned}
$$

Also, multiplying $(7)_{1}$ by $\varphi_{t},(7)_{2}$ by $\psi_{t}$, integrating over $(0,1)$ and adding the two equations, we get

$$
\begin{aligned}
& \rho\left\langle\varphi_{t}, \varphi_{t t}\right\rangle+k\left\langle\varphi_{x}, \varphi_{x t}\right\rangle+J\left\langle\psi_{t}, \psi_{t t}\right\rangle+a\left\langle\psi, \psi_{t}\right\rangle+b \frac{d}{d t}\left\langle\varphi_{x}, \psi\right\rangle+\frac{1}{2} \frac{d}{d t}\left[\left(1-\int_{0}^{t} g(s) d s\right)\left\|\psi_{x}(t)\right\|^{2}\right]+\frac{1}{2} \frac{d}{d t}\left(g \circ \psi_{x}\right)(t) \\
& =\frac{1}{2}\left(g^{\prime} \circ \psi_{x}\right)(t)-\frac{1}{2} g(t)\left\|\psi_{x}(t)\right\|^{2}-\mu_{1}\left\|\psi_{t}(t)\right\|^{2}-\mu_{2}\left\langle\psi_{t}(t), \psi_{t}(t-\tau(t))\right\rangle .
\end{aligned}
$$

Young's inequality yields

$$
-\mu_{2}\left\langle\psi_{t}(t), \psi_{t}(t-\tau(t))\right\rangle \leq \frac{\mu_{1}}{2}\left\|\psi_{t}\right\|^{2}+\frac{\mu_{2}^{2}}{2 \mu_{1}}\left\|\psi_{t}(t-\tau(t))\right\|^{2} .
$$

Substituting (24) into (23) and taking into account (25), assumptions (A3) and (A4), we get

$$
\begin{aligned}
E^{\prime}(t) \leq & \frac{1}{2}\left(g^{\prime} \circ \psi_{x}\right)(t)-\frac{1}{2} g(t)\left\|\psi_{x}(t)\right\|^{2}-\left(\frac{\mu_{1}}{2}-\frac{\zeta}{2}\right)\left\|\psi_{t}\right\|^{2} \\
& -\left[\frac{\zeta}{2} e^{-\lambda \tau_{1}}(1-d)-\frac{\mu_{2}^{2}}{2 \mu_{1}}\right]\left\|\psi_{t}(t-\tau(t))\right\|^{2}-\frac{\lambda \zeta}{2} \int_{t-\tau(t)}^{t} e^{-\lambda(t-s)}\left\|\psi_{t}(s)\right\|^{2} d s .
\end{aligned}
$$

From condition (21), we can select $\zeta>0$ so that

$$
\frac{\mu_{2}^{2} e^{\lambda \tau_{1}}}{\mu_{1}(1-d)}<\zeta<\mu_{1}
$$


Hence, (22) follows from (26) by virtue of $(A 1)-(A 2)$ and (27). This completes the proof.

Lemma 6. Let $t_{0}>0$. Then, the functional $F_{1}(t)=-J \int_{0}^{1} \psi_{t} \int_{0}^{t} g(t-s)(\psi(t)-\psi(s)) d s d x$ along the solution of $(7)$ for any $\epsilon_{1}, \epsilon_{2}>0$ and $0<\alpha<1$ satisfies the estimate

$$
\begin{aligned}
F_{1}^{\prime}(t) \leq & -\frac{J g_{0}}{2}\left\|\psi_{t}\right\|^{2}+\epsilon_{1}\left\|\psi_{x}\right\|^{2}+\epsilon_{2}\left\|\varphi_{x}\right\|^{2}+\epsilon_{3}\|\psi\|^{2}+C\left\|\psi_{t}(t-\tau(t))\right\|^{2} \\
& +C A_{\alpha}\left(1+\frac{1}{\epsilon_{1}}+\frac{1}{\epsilon_{2}}+\frac{1}{\epsilon_{3}}\right)\left(h \circ \psi_{x}\right)(t), \forall t \geq t_{0} .
\end{aligned}
$$

Proof. Differentiating $F_{1}$, using $(7)_{2}$ and integration by parts, we get

$$
\begin{aligned}
F_{1}^{\prime}(t)= & -J\left(\int_{0}^{t} g(s) d s\right)\left\|\psi_{t}\right\|^{2}-\underbrace{J \int_{0}^{1} \psi_{t} \int_{0}^{t} g^{\prime}(t-s)(\psi(t)-\psi(s)) d s d x}_{I_{1}} \\
& +\underbrace{\left(\delta-\int_{0}^{t} g(s) d s\right) \int_{0}^{1} \psi_{x} \int_{0}^{t} g(t-s)\left(\psi_{x}(t)-\psi_{x}(s)\right) d s d x}_{I_{2}} \\
& +\underbrace{\underbrace{1}_{0}\left(\int_{0}^{t} g(t-s)\left(\psi_{x}(t)-\psi_{x}(s)\right) d s\right)^{2} d x}_{I_{5}}+\underbrace{b \int_{0}^{1} \varphi_{x} \int_{0}^{t} g(t-s)(\psi(t)-\psi(s)) d s d x}_{I_{4}} \\
& +\underbrace{a \int_{0}^{1} \psi \int_{0}^{t} g(t-s)(\psi(t)-\psi(s)) d s d x}_{I_{6}}+\underbrace{\mu_{0}^{1} \psi_{t} \int_{0}^{t} g(t-s)(\psi(t)-\psi(s)) d s d x}_{\mu_{1}} \\
& +\underbrace{\psi(s)) d s d x}_{\mu_{2} \int_{0}^{1} \psi_{t}(t-\tau(t)) \int_{0}^{t} g(t-s)(\psi(t)} . \underbrace{\int_{0}} .
\end{aligned}
$$

Using Cauchy-Schwarz, Young's and Poincaré's inequalities, Lemmas 1- 3 and similar computations as in (19), we estimate $I_{1}-I_{7}$ as follows:

$$
\left\{\begin{array}{rlrl}
I_{1}= & J \int_{0}^{1} \psi_{t} \int_{0}^{t} h(t-s)(\psi(t)-\psi(s)) d s d x & & \\
& -J \alpha \int_{0}^{1} \psi_{t} \int_{0}^{t} g(t-s)(\psi(t)-\psi(s)) d s d x & & \\
\leq & \frac{\sigma_{1}}{2}\left\|\psi_{t}\right\|^{2}+\frac{C}{\sigma_{1}} \int_{0}^{1}\left(\int_{0}^{t} h(t-s)(\psi(t)-\psi(s)) d s\right)^{2} d x & & \\
& +\frac{C}{\sigma_{1}} \int_{0}^{1}\left(\int_{0}^{t} g(t-s)(\psi(t)-\psi(s)) d s\right)^{2} d x & \\
\leq & \frac{\sigma_{1}}{2}\left\|\psi_{t}\right\|^{2}+\frac{C}{\sigma_{1}}\left(\int_{0}^{t} h(s) d s\right)(h \circ \psi)(t)+\frac{C A_{\alpha}}{\sigma_{1}}(h \circ \psi)(t) & \\
\leq & \frac{\sigma_{1}}{2}\left\|\psi_{t}\right\|^{2}+\frac{C\left(A_{\alpha}+1\right)}{\sigma_{1}}\left(h \circ \psi_{x}\right)(t), & & \\
I_{2} \leq & \epsilon_{1}\left\|\psi_{x}\right\|^{2}+\frac{C A_{\alpha}}{\epsilon_{1}}\left(h \circ \psi_{x}\right)(t), & & \text { for any } \sigma_{1}>0 \\
I_{3} \leq & A_{\alpha}\left(h \circ \psi_{x}\right)(t), & & \text { for any } \epsilon_{1}>0 \\
I_{4} \leq & \epsilon_{2}\left\|\varphi_{x}\right\|^{2}+\frac{C A_{\alpha}}{\epsilon_{2}}\left(h \circ \psi_{x}\right)(t), & & \text { for any } \epsilon_{2}>0 \\
I_{5} \leq & \epsilon_{3}\|\psi\|^{2}+\frac{C A_{\alpha}}{\epsilon_{3}}\left(h \circ \psi_{x}\right)(t), & & \text { for any } \epsilon_{3}>0 \\
I_{6} \leq & \frac{\sigma_{1}}{2}\left\|\psi_{t}\right\|^{2}+\frac{C A_{\alpha}}{\sigma_{1}}\left(h \circ \psi_{x}\right)(t), & & \text { for any } \sigma_{1}>0 \\
I_{7} \leq & \frac{\sigma_{1}}{2}\left\|\psi_{t}(t-\tau(t))\right\|^{2}+\frac{C A_{\alpha}}{\sigma_{1}}\left(h \circ \psi_{x}\right)(t), & & \text { for any } \sigma_{1}>0 .
\end{array}\right.
$$


Substituting the estimates in (30) into (29), we arrive at

$$
\begin{aligned}
F_{1}^{\prime}(t) \leq- & \left(J \int_{0}^{t} g(s) d s-\sigma_{1}\right)\left\|\psi_{t}\right\|^{2}+\epsilon_{1}\left\|\psi_{x}\right\|^{2}+\epsilon_{2}\left\|\varphi_{x}\right\|^{2}+\epsilon_{3}\|\psi\|^{2} \\
& +C A_{\alpha}\left(1+\frac{1}{\sigma_{1}}+\frac{1}{\epsilon_{1}}+\frac{1}{\epsilon_{2}}+\frac{1}{\epsilon_{3}}\right)\left(h \circ \psi_{x}\right)(t)+\frac{\sigma_{1}}{2}\left\|\psi_{t}(t-\tau(t))\right\|^{2} .
\end{aligned}
$$

From $(A 1)$, we have that $g(0)>0$ and $g$ is continuous. Therefore, for $t \geq t_{0}>0$, we obtain

$$
\int_{0}^{t} g(s) d s \geq \int_{0}^{t_{0}} g(s) d s=g_{0}>0
$$

Thus, we select $\sigma_{1}=\frac{J g_{0}}{2}$ to get (28). This completes the proof.

Lemma 7. Let $(\varphi, \psi)$ be the solution of Problem (7). Then, the functional $F_{2}(t)=-\rho \int_{0}^{1} \varphi_{t} \varphi d x$ satisfies the estimate

$$
F_{2}^{\prime}(t) \leq-\rho\left\|\varphi_{t}\right\|^{2}+C\left\|\varphi_{x}\right\|^{2}+C\left\|\psi_{x}\right\|^{2}, \forall t \geq 0
$$

Proof. Differentiation of $F_{2}$, using $(7)_{1}$ and integration by parts, we obtain

$$
F_{2}^{\prime}(t)=-\rho\left\|\varphi_{t}\right\|^{2}+k\left\|\varphi_{x}\right\|^{2}+b \int_{0}^{1} \varphi_{x} \psi d x .
$$

Applying Young's and Poincaré's inequalities, we obtain (33). This completes the proof.

Lemma 8. The functional $F_{3}(t)=J \int_{0}^{1} \psi_{t} \psi d x+\frac{b \rho}{k} \int_{0}^{1} \psi \int_{0}^{x} \varphi_{t}(y) d y d x$ along the solution of problem (7) for any $\epsilon_{4}>0$ and $0<\alpha<1$ satisfies the estimate

$$
\begin{aligned}
F_{3}^{\prime}(t)= & -\frac{l}{2}\left\|\psi_{x}\right\|^{2}-\left(a-\frac{b^{2}}{k}\right)\|\psi\|^{2}+\epsilon_{4}\left\|\varphi_{t}\right\|^{2}++C\left(1+\frac{1}{\epsilon_{4}}\right)\left\|\psi_{t}\right\|^{2} \\
& +C A_{\alpha}\left(h \circ \psi_{x}\right)(t)+C\left\|\psi_{t}(t-\tau(t))\right\|^{2}, \forall t \geq 0 .
\end{aligned}
$$

Proof. Differentiation of $F_{3}$, using (7) and integration by parts leads to

$$
\begin{aligned}
F_{3}^{\prime}(t)= & J\left\|\psi_{t}\right\|^{2}-\delta\left\|\psi_{x}\right\|^{2}-\left(a-\frac{b^{2}}{k}\right)\|\psi\|^{2}+\underbrace{\frac{b \rho}{k} \int_{0}^{1} \psi_{t} \int_{0}^{x} \varphi_{t}(y) d y d x}_{I_{8}} \\
& +\underbrace{\int_{0}^{1} \psi_{x} \int_{0}^{t} g(t-s) \psi_{x}(s) d s d x}_{I_{9}}-\underbrace{\mu_{1} \int_{0}^{1} \psi \psi_{t} d x}_{I_{10}}-\underbrace{\mu_{2} \int_{0}^{1} \psi \psi_{t}(t-\tau(t)) d x}_{I_{11}} .
\end{aligned}
$$

Using Cauchy-Schwarz, Young's and Poincaré's inequalities together with Lemmas 1-3, we have

$$
\begin{aligned}
I_{8} & \leq \epsilon_{4} \int_{0}^{1}\left(\int_{0}^{x} \varphi_{t}(y) d y\right)^{2} d x+\frac{(b \rho)^{2}}{4 k^{2} \epsilon_{4}}\left\|\psi_{t}\right\|^{2} \\
& \leq \epsilon_{4}\left\|\varphi_{t}\right\|^{2}+\frac{(b \rho)^{2}}{4 k^{2} \epsilon_{4}}\left\|\psi_{t}\right\|^{2}, \text { for any } \epsilon_{4}>0, \\
I_{9} & =\int_{0}^{1} \psi_{x} \int_{0}^{t} g(t-s)\left(\psi_{x}(s)-\psi_{x}(t)\right) d s d x+\left(\int_{0}^{t} g(s) d s\right)\left\|\psi_{x}\right\|^{2} \\
& \leq\left(\int_{0}^{t} g(s) d s+\frac{\sigma_{2}}{3}\right)\left\|\psi_{x}\right\|^{2}+\frac{C}{\sigma_{2}} \int_{0}^{1}\left(\int_{0}^{t} g(t-s)\left(\psi_{x}(s)-\psi_{x}(t)\right) d s\right)^{2} d x \\
& \leq\left(\int_{0}^{t} g(s) d s+\frac{\sigma_{2}}{3}\right)\left\|\psi_{x}\right\|^{2}+\frac{C A_{\alpha}}{\sigma_{2}}\left(h \circ \psi_{x}\right)(t), \text { for any } \sigma_{2}>0, \\
I_{10} & \leq \frac{\sigma_{2}}{3}\left\|\psi_{x}\right\|^{2}+\frac{C \mu_{1}^{2}}{\sigma_{2}}\left\|\psi_{t}\right\|^{2}, \text { for any } \sigma_{2}>0,
\end{aligned}
$$


and

$$
I_{11} \leq \frac{\sigma_{2}}{3}\left\|\psi_{x}\right\|^{2}+\frac{C \mu_{2}^{2}}{\sigma_{2}}\left\|\psi_{t}(t-\tau(t))\right\|^{2}, \text { for any } \sigma_{2}>0 .
$$

Substituting (36)-(39) into (35), we arrive at

$$
\begin{aligned}
F_{3}^{\prime}(t)= & -\left(\delta-\int_{0}^{t} g(s) d s-\sigma_{2}\right)\left\|\psi_{x}\right\|^{2}-\left(a-\frac{b^{2}}{k}\right)\|\psi\|^{2}+\epsilon_{4}\left\|\varphi_{t}\right\|^{2} \\
& +\left(J+\frac{(b \rho)^{2}}{4 k^{2} \epsilon_{4}}+\frac{C \mu_{1}^{2}}{\sigma_{2}}\right)\left\|\psi_{t}\right\|^{2}+\frac{C A_{\alpha}}{\sigma_{2}}\left(h \circ \psi_{x}\right)(t)+\frac{C \mu_{2}^{2}}{\sigma_{2}}\left\|\psi_{t}(t-\tau(t))\right\|^{2} .
\end{aligned}
$$

We choose $\sigma_{2}=\frac{l}{2}$ to obtain (34). This completes the proof.

Lemma 9. Assume $\frac{k}{\rho}=\frac{\delta}{J}$. Then, the functional

$$
F_{4}(t)=\frac{|b| \delta \rho}{b k} \int_{0}^{1} \varphi_{t} \psi_{x} d x+\frac{|b| J}{b} \int_{0}^{1} \psi_{t} \varphi_{x} d x-\frac{|b| \rho}{b k} \int_{0}^{1} \varphi_{t} \int_{0}^{t} g(t-s) \psi_{x}(s) d s d x,
$$

along the solution of (7), for any $\epsilon_{5}>0$ and $0<\alpha<1$ satisfies the estimate

$$
\begin{aligned}
F_{4}^{\prime}(t) \leq & -\frac{|b|}{2}\left\|\varphi_{x}\right\|^{2}+\epsilon_{5}\left\|\varphi_{t}\right\|^{2}+C\left(1+\frac{1}{\epsilon_{5}}\right)\left\|\psi_{x}\right\|^{2}+C\left\|\psi_{t}\right\|^{2}+C\left\|\psi_{t}(t-\tau(t))\right\|^{2} \\
& +\frac{C\left(A_{\alpha}+1\right)}{\epsilon_{5}}\left(h \circ \psi_{x}\right)(t), \forall t \geq 0 .
\end{aligned}
$$

Proof. Differentiating $F_{4}$, we get

$$
\begin{aligned}
F_{4}^{\prime}(t)= & \frac{|b| \delta \rho}{b k} \int_{0}^{1} \varphi_{t t} \psi_{x} d x+\frac{|b| \delta \rho}{b k} \int_{0}^{1} \varphi_{t} \psi_{x t} d x+\frac{|b| J}{b} \int_{0}^{1} \psi_{t t} \varphi_{x} d x \\
& +\frac{|b| J}{b} \int_{0}^{1} \psi_{t} \varphi_{x t} d x-\frac{|b| \rho}{b k} \int_{0}^{1} \varphi_{t t} \int_{0}^{t} g(t-s) \psi_{x}(s) d s d x \\
& -\frac{|b| \rho}{b k} \int_{0}^{1} \varphi_{t} \int_{0}^{t} g^{\prime}(t-s) \psi_{x}(s) d s d x-\frac{|b| \rho}{b k} g(0) \int_{0}^{1} \varphi_{t} \psi_{x} d x
\end{aligned}
$$

Using these equations in (7) and integration by parts, we arrive at

$$
\begin{aligned}
F_{4}^{\prime}(t)= & \frac{|b| \delta \rho}{k}\left\|\psi_{x}\right\|^{2}+\frac{|b|}{b}\left(\frac{\delta \rho}{k}-J\right) \int_{0}^{1} \varphi_{x} \psi_{x t} d x-|b|\left\|\varphi_{x}\right\|^{2} \\
& -\frac{|b| \mu_{1}}{b} \int_{0}^{1} \psi_{t} \varphi_{x} d x-\frac{|b| \mu_{2}}{b} \int_{0}^{1} \psi_{t}(t-\tau(t)) \varphi_{x} d x \\
& -\frac{|b| a}{b} \int_{0}^{1} \psi \varphi_{x} d x-\frac{|b|}{k} \int_{0}^{1} \psi_{x} \int_{0}^{t} g(t-s) \psi_{x}(s) d s d x \\
& -\frac{|b| \rho}{b k} \int_{0}^{1} \varphi_{t} \int_{0}^{t} g^{\prime}(t-s) \psi_{x}(s) d s d x-\frac{|b| \rho}{b k} g(0) \int_{0}^{1} \varphi_{t} \psi_{x} d x
\end{aligned}
$$

Using the fact that $\frac{k}{\rho}=\frac{\delta}{j}$, we get

$$
\begin{aligned}
F_{4}^{\prime}(t)= & -|b|\left\|\varphi_{x}\right\|^{2}+\frac{|b| \delta \rho}{k}\left\|\psi_{x}\right\|^{2}-\underbrace{\frac{|b| a}{b} \int_{0}^{1} \psi \varphi_{x} d x}_{G_{1}}-\underbrace{\frac{|b| \mu_{1}}{b} \int_{0}^{1} \psi_{t} \varphi_{x} d x}_{G_{2}} \\
& -\underbrace{\frac{|b| \mu_{2}}{b} \int_{0}^{1} \psi_{t}(t-\tau(t)) \varphi_{x} d x}_{G_{3}}-\underbrace{\frac{|b|}{k} \int_{0}^{1} \psi_{x} \int_{0}^{t} g(t-s) \psi_{x}(s) d s d x}_{G_{5}} \\
& -\underbrace{\frac{|b| \rho}{b k} \int_{0}^{1} \varphi_{t} \int_{0}^{t} g^{\prime}(t-s) \psi_{x}(s) d s d x}_{G_{4}}-\underbrace{\frac{|b| \rho}{b k} g(0) \int_{0}^{1} \varphi_{t} \psi_{x} d x}_{G_{6}} .
\end{aligned}
$$


Applying Cauchy-Schwarz, Young's and Poincaré's inequalities, taking into account Lemmas $1-3, h=\alpha g-$ $g^{\prime}$, we have for any $\sigma_{3}, \epsilon_{5}>0$

$$
\begin{aligned}
G_{1} \leq & \frac{\sigma_{3}}{3}\left\|\varphi_{x}\right\|^{2}+\frac{C}{\sigma_{3}}\left\|\psi_{x}\right\|^{2}, \\
G_{2} \leq & \frac{\sigma_{3}}{3}\left\|\varphi_{x}\right\|^{2}+\frac{C}{\sigma_{3}}\left\|\psi_{t}\right\|^{2}, \\
G_{3} \leq & \frac{\sigma_{3}}{3}\left\|\varphi_{x}\right\|^{2}+\frac{C}{\sigma_{3}}\left\|\psi_{t}(t-\tau(t))\right\|^{2}, \\
G_{4} \leq & \frac{|b|}{2 k}\left\|\psi_{x}\right\|^{2}+\frac{|b|}{2 k} \int_{0}^{1}\left(\int_{0}^{t} g(t-s) \psi_{x}(s)-\psi_{x}(t) d s+\int_{0}^{t} g(s) d s \psi_{x}(t)\right)^{2} d x \\
\leq & \frac{|b|}{2 k}\left\|\psi_{x}\right\|^{2}+\frac{|b|}{k} \int_{0}^{1}\left(\int_{0}^{t} g(t-s) \psi_{x}(s)-\psi_{x}(t) d s\right)^{2} d x+\frac{|b|(\delta-l)^{2}}{2 k}\left\|\psi_{x}\right\|^{2} \\
\leq & \left(\frac{|b|}{2 k}+\frac{|b|(\delta-l)^{2}}{k}\right)\left\|\psi_{x}\right\|^{2}+\frac{|b|}{k} A_{\alpha}\left(h \circ \psi_{x}\right)(t), \\
G_{5}= & \frac{|b| \rho}{b k} \int_{0}^{1} \varphi_{t} \int_{0}^{t} h(t-s)\left(\psi_{x}(s)-\psi_{x}(t)\right) d s d x+\frac{|b| \rho}{b k}\left(\int_{0}^{t} h(s) d s\right) \int_{0}^{1} \varphi_{t} \psi_{x} d x \\
& -\frac{|b| \rho \alpha}{b k} \int_{0}^{1} \varphi_{t} \int_{0}^{t} g(t-s) \psi_{x}(s) d s d x \\
\leq & \frac{3 \epsilon_{5}}{4}\left\|\varphi_{t}\right\|^{2}+\frac{C}{\epsilon_{5}} \int_{0}^{1}\left(\int_{0}^{t} h(t-s) \psi_{x}(s)-\psi_{x}(t) d s\right)^{2} d x+\frac{C}{\epsilon_{5}}\left\|\psi_{x}\right\|^{2} \\
& +\frac{C}{\epsilon_{5}} \int_{0}^{1}\left(\int_{0}^{t} g(t-s) \psi_{x}(s)-\psi_{x}(t) d s+\int_{0}^{t} g(s) d s \psi_{x}(t)\right)^{2} d x \\
\leq & \frac{3 \epsilon_{5}}{4}\left\|\varphi_{t}\right\|^{2}+\frac{C}{\epsilon_{5}}\left\|\psi_{x}\right\|^{2}+\frac{C\left(A_{\alpha}+1\right)}{\epsilon_{5}}\left(h \circ \psi_{x}\right)(t),
\end{aligned}
$$

and

$$
G_{6} \leq \frac{\epsilon_{5}}{4}\left\|\varphi_{t}\right\|^{2}+\frac{C}{\epsilon_{5}}\left\|\psi_{x}\right\|^{2}
$$

Substitution of (43) - (48) into (42) yields

$$
\begin{aligned}
F_{4}^{\prime}(t)= & -\left(|b|-\sigma_{3}\right)\left\|\varphi_{x}\right\|^{2}+\left(\frac{|b| \delta \rho}{k}+\frac{C}{\sigma_{3}}+\frac{|b|}{2 k}+\frac{|b|(\delta-l)^{2}}{k}+\frac{C}{\epsilon_{5}}\right)\left\|\psi_{x}\right\|^{2} \\
& +\epsilon_{5}\left\|\varphi_{t}\right\|^{2}+\frac{C}{\sigma_{3}}\left\|\psi_{t}\right\|^{2}+\frac{C}{\sigma_{3}}\left\|\psi_{t}(t-\tau(t))\right\|^{2}+\left(C A_{\alpha}+\frac{C\left(A_{\alpha}+1\right)}{\epsilon_{5}}\right)\left(h \circ \psi_{x}\right)(t) .
\end{aligned}
$$

Finally, we choose $\sigma_{3}=\frac{|b|}{2}$ to obtain (41). This completes the proof.

Lemma 10. The functional $F_{5}(t)=\int_{0}^{t} f(t-s)\left\|\psi_{x}(s)\right\|^{2} d s$, where $f(t)=\int_{t}^{+\infty} g(s) d s$, along the solution of (7) satisfies the estimate

$$
F_{5}^{\prime}(t) \leq 3(\delta-l)\left\|\psi_{x}\right\|_{2}^{2}-\frac{1}{2}\left(g \circ \psi_{x}\right)(t), \forall t \geq 0
$$

Proof. Differentiation of $F_{5}$ and using the fact that $f^{\prime}(t)=-g(t)$ lead to

$$
\begin{aligned}
F_{5}^{\prime}(t) & =\int_{0}^{t} f^{\prime}(t-s)\left\|\psi_{x}(s)\right\|^{2} d s+f(0)\left\|\psi_{x}\right\|^{2} \\
& =-\left(g \circ \psi_{x}\right)(t)+f(t)\left\|\psi_{x}\right\|^{2}-2 \int_{0}^{1} \psi_{x} \int_{0}^{t} g(t-s)\left(\psi_{x}(s)-\psi_{x}(t)\right) d s d x
\end{aligned}
$$

Cauchy-Schwarz inequality and condition $(A 1)$ give $-2 \int_{0}^{1} \psi_{x} \int_{0}^{t} g(t-s)\left(\psi_{x}(s)-\psi_{x}(t)\right) d s d x \leq 2(\delta-l)\left\|\psi_{x}\right\|^{2}+\frac{\int_{0}^{t} g(s) d s}{2(\delta-l)}\left(g \circ \psi_{x}\right)(t) \leq 2(\delta-l)\left\|\psi_{x}\right\|^{2}+\frac{1}{2}\left(g \circ \psi_{x}\right)(t)$. 
Therefore,

$$
F_{5}^{\prime}(t) \leq 2(\delta-l)\left\|\psi_{x}\right\|^{2}-\frac{1}{2}\left(g \circ \psi_{x}\right)(t)+f(t)\left\|\psi_{x}\right\|^{2} .
$$

Since $f^{\prime}(t)=-g(t) \leq 0$, it follows that $f(t) \leq f(0)=\delta-l$. Thus, we have

$$
F_{5}^{\prime}(t) \leq 3(\delta-l)\left\|\psi_{x}\right\|^{2}-\frac{1}{2}\left(g \circ \psi_{x}\right)(t), \forall t \geq 0 .
$$

For the next lemma, we consider the Lyapunov functional $K$ defined by

$$
K(t)=N E(t)+N_{1} F_{1}(t)+N_{2} F_{2}(t)+N_{3} F_{3}(t)+N_{4} F_{4}(t),
$$

where $N, N_{j}, j=1,2,3,4$ are positive constants to be specified later.

Lemma 11. Assume $\frac{k}{\rho}=\frac{\delta}{J}$. Then, for suitable choice of $N, N_{j}, j=1,2,3,4$, the Lyapunov functional $K$ along the solution of (7) satisfies the estimate

$$
K^{\prime}(t) \leq-\beta\left(\left\|\varphi_{t}\right\|^{2}+\left\|\varphi_{x}\right\|^{2}+\left\|\psi_{t}\right\|^{2}+\left\|\psi_{x}\right\|^{2}+\|\psi\|^{2}\right)+\frac{1}{4}\left(g \circ \psi_{x}\right)(t), \forall t \geq t_{0}
$$

for some $\beta>0$ and $K \sim E$, that is

$$
\alpha_{1} E(t) \leq K(t) \leq \alpha_{2} E(t), \forall t \geq 0
$$

holds for some $\alpha_{1}, \alpha_{2}>0$.

Proof. Using (51) and recalling that $h(t)=\alpha g(t)-g^{\prime}(t)$, then Lemmas 6-10 yields, for all $t \geq t_{0}$,

$$
\begin{aligned}
K^{\prime}(t) \leq & -\left[N_{2} \rho-N_{3} \epsilon_{4}-N_{4} \epsilon_{5}\right]\left\|\varphi_{t}\right\|^{2}-\left[\frac{N_{4}|b|}{2}-N_{2} C-N_{1} \epsilon_{2}\right]\left\|\varphi_{x}\right\|^{2} \\
& -\left[N \gamma_{2}+\frac{N_{1} J g_{0}}{2}-N_{3}\left(1+\frac{1}{\epsilon_{4}}\right)-N_{4} C\right]\left\|\psi_{t}\right\|^{2} \\
& -\left[\frac{N_{3} l}{2}-N_{2} C-N_{1} \epsilon_{1}-N_{4} C\left(1+\frac{1}{\epsilon_{5}}\right)\right]\left\|\psi_{x}\right\|^{2}-\left[N_{3} \gamma_{1}-N_{1} \epsilon_{3}\right]\|\psi\|^{2}+\frac{N \alpha}{2}\left(g \circ \psi_{x}\right)(t) \\
& -\left[N \gamma_{3}-N_{1} C-N_{3} C-N_{4} C\right]\left\|\psi_{t}(t-\tau(t))\right\|^{2}-\frac{N \lambda \zeta}{2} \int_{t-\tau(t)}^{t} e^{-\lambda(t-s)\left\|\psi_{t}(s)\right\|^{2} d s} \\
& -\left[\frac{N}{2}-C A_{\alpha}\left(N_{1}\left(1+\frac{1}{\epsilon_{1}}+\frac{1}{\epsilon_{2}}+\frac{1}{\epsilon_{3}}\right)+N_{3}+\frac{N_{4}}{\epsilon_{5}}\right)\right]\left(h \circ \psi_{x}\right)(t),
\end{aligned}
$$

where $\gamma_{1}=\left(a-\frac{b^{2}}{k}\right)>0, \gamma_{2}=\left(\frac{\mu_{1}}{2}-\frac{\zeta}{2}\right)>0, \gamma_{3}=\left(\frac{\zeta}{2} e^{-\lambda \tau_{1}}(1-d)-\frac{\mu_{2}^{2}}{2 \mu_{1}}\right)>0$ by virtue of (3) and (27). Next, we choose

$$
N_{2}=1, \epsilon_{1}=\frac{N_{3} l}{4 N_{1}}, \epsilon_{2}=\frac{N_{4}|b|}{4 N_{1}}, \epsilon_{3}=\frac{N_{3} \gamma_{1}}{2 N_{2}}, \epsilon_{4}=\frac{\rho}{4 N_{3}}, \epsilon_{5}=\frac{\rho}{4 N_{4}}
$$

and (54) becomes

$$
\begin{aligned}
K^{\prime}(t) \leq & -\frac{\rho}{2}\left\|\varphi_{t}\right\|^{2}-\left[\frac{N_{4}|b|}{4}-C\right]\left\|\varphi_{x}\right\|^{2}-\left[N \gamma_{2}+\frac{N_{1} J g_{0}}{2}-N_{3}\left(1+\frac{4 N_{3}}{\rho}\right)-N_{4} C\right]\left\|\psi_{t}\right\|^{2} \\
& -\left[\frac{N_{3} l}{4}-N_{4} C\left(1+\frac{4 N_{4}}{\rho}\right)-C\right]\left\|\psi_{x}\right\|^{2}-\frac{N_{3} \gamma_{1}}{2}\|\psi\|^{2} \\
& -\left[N \gamma_{3}-\left(N_{1}+N_{3}+N_{4}\right) C\right]\left\|\psi_{t}(t-\tau(t))\right\|^{2} \\
& +\frac{N \alpha}{2}\left(g \circ \psi_{x}\right)(t)-\frac{N \lambda \zeta}{2} \int_{t-\tau(t)}^{t} e^{-\lambda(t-s)}\left\|\psi_{t}(s)\right\|^{2} d s \\
& -\left[\frac{N}{2}-C A_{\alpha}\left(N_{1}\left(1+\frac{4 N_{1}}{N_{3} l}+\frac{4 N_{1}}{N_{4}|b|}+\frac{2 N_{1}}{N_{3} \gamma_{1}}\right)+N_{3}+\frac{N_{4}^{2}}{\rho}\right)\right]\left(h \circ \psi_{x}\right)(t) .
\end{aligned}
$$


Now, we choose the remaining constants: First, we select $N_{4}$ so that

$$
\frac{N_{4}|b|}{4}-C>0
$$

Then, we choose $N_{3}$ large enough such that

$$
\frac{N_{3} l}{4}-N_{4} C\left(1+\frac{4 N_{4}}{\rho}\right)-C>0 .
$$

Hence $N_{3}$ and $N_{4}$ are fixed, we choose $N_{1}$ large so that

$$
\frac{N_{1} J g_{0}}{2}-N_{3}\left(1+\frac{4 N_{3}}{\rho}\right)-N_{4} C>0 .
$$

We have that $\frac{\alpha g^{2}(s)}{h(s)}=\frac{\alpha g^{2}(s)}{\alpha g(s)-g^{\prime}(s)}<g(s)$. Thus, using the dominated convergence theorem, we get

$$
\lim _{\alpha \rightarrow 0} \alpha A_{\alpha}=\lim _{\alpha \rightarrow 0} \int_{0}^{+\infty} \frac{\alpha g^{2}(s)}{\alpha g(s)-g^{\prime}(s)} d s=0 .
$$

Thus, there exist $0<\alpha_{0}<1$ such that for all $0<\alpha \leq \alpha_{0}$, we have

$$
\alpha A_{\alpha}<\frac{1}{4 C\left(N_{1}\left(1+\frac{4 N_{1}}{N_{3} l}+\frac{4 N_{1}}{N_{4}|b|}+\frac{2 N_{1}}{N_{3} \gamma_{1}}\right)+N_{3}+\frac{N_{4}^{2}}{\rho}\right)} .
$$

Finally, we choose $N$ so large and take $\alpha=\frac{1}{2 N}$ Such that

$$
\begin{aligned}
& N \gamma_{3}-\left(N_{1}+N_{3}+N_{4}\right) C>0, \\
& \frac{N}{2}-C A_{\alpha}\left(N_{1}\left(1+\frac{4 N_{1}}{N_{3} l}+\frac{4 N_{1}}{N_{4}|b|}+\frac{2 N_{1}}{N_{3} \gamma_{1}}\right)+N_{3}+\frac{N_{4}^{2}}{\rho}\right)>0 .
\end{aligned}
$$

The analysis from (55) - (61) yields (52). Applying Young's, Cauchy-Schwarz, and Poincaré's inequalities, we obtain (53) easily. This completes the proof.

\section{Main stability result}

The main stability result of the work is the following:

Theorem 2. Assume $\frac{k}{\rho}=\frac{\delta}{J}$ and $(A 1)-(A 5)$ hold. Then, there exist $\lambda_{1}>0, \lambda_{2}>0$ such that the solution energy (20) satisfies

$$
E(t) \leq \lambda_{2} M_{1}^{-1}\left(\lambda_{1} \int_{t_{0}}^{t} \xi(s) d s\right), \forall t \geq t_{0}
$$

where $M_{1}(t)=\int_{t}^{r} \frac{1}{s M^{\prime}(s)} d s$ and $M_{1}$ is a strictly decreasing and strictly convex function on $(0, r]$ with $\lim _{t \rightarrow 0} M_{1}(t)=$ $+\infty$.

Proof. From (13) and (22), we have $\forall t \geq t_{0}$

$$
\int_{0}^{t_{0}} g(s)\left\|\psi_{x}(t)-\psi_{x}(t-s)\right\|^{2} d s \leq-\frac{g(0)}{\beta_{1}} \int_{0}^{t_{0}} g^{\prime}(s)\left\|\psi_{x}(t)-\psi_{x}(t-s)\right\|^{2} d s \leq-C E^{\prime}(t) .
$$

Thus, (52) and (63) implies

$$
K^{\prime}(t) \leq-\eta E(t)+\frac{1}{2}\left(g \circ \psi_{x}\right)(t) \leq-\eta E(t)-C E^{\prime}(t)+\frac{1}{2} \int_{t_{0}}^{t} g(s)\left\|\psi_{x}(t)-\psi_{x}(t-s)\right\|^{2} d s,
$$

for some $\eta>0$. Let $K_{1}=K+C E \sim E$, by virtue of (53), it follows that

$$
K_{1}^{\prime}(t) \leq-\eta E(t)+\frac{1}{2} \int_{t_{0}}^{t} g(s)\left\|\psi_{x}(t)-\psi_{x}(t-s)\right\|^{2} d s, \forall t \geq t_{0}
$$


To complete the proof, we divide it into two parts:

Case 1: when $M(t)$ is linear. Multiplying (64) by $\xi(t)$, keeping in mind (22) and (A2), we get

$$
\begin{aligned}
\xi(t) K_{1}^{\prime}(t) & \leq-\eta \xi(t) E(t)+\frac{1}{2} \xi(t) \int_{t_{0}}^{t} g(s)\left\|\psi_{x}(t)-\psi_{x}(t-s)\right\|^{2} d s \\
& \leq-\eta \xi(t) E(t)+\frac{1}{2} \int_{t_{0}}^{t} \xi(s) g(s)\left\|\psi_{x}(t)-\psi_{x}(t-s)\right\|^{2} d s \\
& \leq-\eta \xi(t) E(t)-\frac{1}{2} \int_{t_{0}}^{t} g^{\prime}(s)\left\|\psi_{x}(t)-\psi_{x}(t-s)\right\|^{2} d s \\
& \leq-\eta \xi(t) E(t)-C E^{\prime}(t), \forall t \geq t_{0} .
\end{aligned}
$$

From (A2), $\xi$ is non-increasing, thus, we get

$$
\left(\xi K_{1}+C E\right)^{\prime}(t) \leq-\eta \xi(t) E(t), \quad \forall t \geq t_{0}
$$

and

$$
\xi K_{1}+C E \sim E .
$$

Since $K \sim E$, thus, setting $K_{2}(t)=\xi(t) K_{1}(t)+C E(t)$, there exists $\eta_{1}>0$ so such that

$$
K_{2}^{\prime}(t) \leq-\eta \xi(t) E(t) \leq-\eta_{1} \xi(t) K_{2}(t), \quad \forall t \geq t_{0} .
$$

Integration of (68) over $\left(t_{0}, t\right)$ and recalling (67), we obtain

$$
E(t) \leq \lambda_{1} e^{-\lambda_{2} \int_{t_{0}}^{t} \xi(s) d s}=\lambda_{1} M_{1}^{-1}\left(\lambda_{2} \int_{t_{0}}^{t} \xi(s) d s\right) .
$$

Case 2: when $M(t)$ is nonlinear. In this case, we consider $\mathcal{K}(t)=K(t)+F_{5}(t)$. Then, Lemmas 10 and (52) yield for some $d>0$

$$
\mathcal{K}^{\prime}(t) \leq-d E(t), \quad \forall t \geq t_{0}
$$

It follows that $d \int_{t_{0}}^{t} E(s) d s \leq \mathcal{K}\left(t_{0}\right)-\mathcal{K}(t) \leq \mathcal{K}\left(t_{0}\right)$, from which we get

$$
\int_{0}^{+\infty} E(s) d s<\infty .
$$

From (70), we can define $p(t)$ by $p(t):=\omega \int_{t_{0}}^{t}\left\|\psi_{x}(t)-\psi_{x}(t-s)\right\|^{2} d s$, where $0<\omega<1$ so that

$$
p(t)<1, \forall t \geq t_{0} .
$$

Furthermore, we assume $p(t)>0$ for all $t \geq t_{0}$; otherwise, it follows from (64) that the solution energy is exponentially stable. Also, we define the functional $q(t)$ by $q(t)=-\int_{t_{0}}^{t} g^{\prime}(s)\left\|\psi_{x}(t)-\psi_{x}(t-s)\right\|^{2} d s$ and it's easy to see that $q(t) \leq-C E^{\prime}(t), \forall t \geq t_{0}$. From $\left(A_{2}\right), M$ is strictly convex on $(0, r]$ (where $r=g\left(t_{0}\right)$ ) and $M(0)=0$, this implies

$$
M(\theta s) \leq \theta M(s), \quad 0 \leq \theta \leq 1 \text { and } s \in(0, r] .
$$

Using (71),(72), assumption (A2) and Jensen's inequality, we have

$$
\begin{aligned}
q(t) & =\frac{1}{\omega p(t)} \int_{t_{0}}^{t} p(t)\left(-g^{\prime}(s)\right) \omega\left\|\psi_{x}(t)-\psi_{x}(t-s)\right\|^{2} d s \\
& \geq \frac{1}{\omega p(t)} \int_{t_{0}}^{t} p(t) \xi(s) M(g(s)) \omega\left\|\psi_{x}(t)-\psi_{x}(t-s)\right\|^{2} d s \\
& \geq \frac{\xi(t)}{\omega p(t)} \int_{t_{0}}^{t} M(p(t) g(s)) \omega\left\|\psi_{x}(t)-\psi_{x}(t-s)\right\|^{2} d s \\
& \geq \frac{\xi(t)}{\omega} M\left(\omega \int_{t_{0}}^{t} g(s)\left\|\psi_{x}(t)-\psi_{x}(t-s)\right\| s^{2} d s\right)
\end{aligned}
$$




$$
=\frac{\xi(t)}{\omega} \bar{M}\left(\omega \int_{t_{0}}^{t} g(s)\left\|\psi_{x}(t)-\psi_{x}(t-s)\right\|^{2} d s\right),
$$

where $\bar{M}$ is the convex extension of $M$ on $(0,+\infty)$, see (14). From (73), we have

$$
\int_{t_{0}}^{t} g(s)\left\|\psi_{x}(t)-\psi_{x}(t-s)\right\|^{2} d s \leq \frac{1}{\omega} \bar{M}^{-1}\left(\frac{\omega q(t)}{\xi(t)}\right) .
$$

Thus, (64) gives

$$
K_{1}^{\prime}(t) \leq-\eta E(t)+C \bar{M}^{-1}\left(\frac{\omega q(t)}{\xi(t)}\right), \forall t \geq t_{0}
$$

Let $r_{0}<r$ to be specified later and define the functional $K_{3}$ by $K_{3}(t):=\bar{M}^{\prime}\left(r_{0} \frac{E(t)}{E(0)}\right) K_{1}(t)+E(t) \sim E(t)$. Since $K_{1} \sim E$. Using (74) and recalling that $E^{\prime}(t) \leq 0, \bar{M}^{\prime}(t)>0, \bar{M}^{\prime \prime}(t)>0$, we have for all $t \geq t_{0}$

$$
\begin{aligned}
K_{3}^{\prime}(t) & =r_{0} \frac{E^{\prime}(t)}{E(0)} \bar{M}^{\prime \prime}\left(r_{0} \frac{E(t)}{E(0)}\right) K_{1}(t)+\bar{M}^{\prime}\left(r_{0} \frac{E(t)}{E(0)}\right) K_{1}^{\prime}(t)+E^{\prime}(t) \\
& \leq-\eta E(t) \bar{M}^{\prime}\left(r_{0} \frac{E(t)}{E(0)}\right)+C \bar{M}^{\prime}\left(r_{0} \frac{E(t)}{E(0)}\right) \bar{M}^{-1}\left(\omega \frac{q(t)}{\xi(t)}\right)+E^{\prime}(t) .
\end{aligned}
$$

Now, we consider conjugate of $\bar{M}$ denoted by $\bar{M}^{*}$ define in the sense of Young, see Arnold [29] page 61-64, define by

$$
\bar{M}^{*}(s)=s\left(\bar{M}^{\prime}\right)^{-1}(s)-\bar{M}\left[\left(\bar{M}^{\prime}\right)(s)\right]
$$

and $\bar{M}^{*}$ satisfies the Young's inequality

$$
X Y \leq \bar{M}^{*}(X)+\bar{M}(Y)
$$

Setting $X=\bar{M}^{\prime}\left(r_{0} \frac{E(t)}{E(0)}\right)$ and $Y=\bar{M}^{-1}\left(\omega^{\frac{q(t)}{\zeta(t)}}\right)$, then (22) and (75)-(77) yield for all $t \geq t_{0}$

$$
\begin{aligned}
K_{3}^{\prime}(t) & \leq-\eta E(t) \bar{M}^{\prime}\left(r_{0} \frac{E(t)}{E(0)}\right)+C \bar{M}^{*}\left(\bar{M}^{\prime}\left(r_{0} \frac{E(t)}{E(0)}\right)\right)+C \omega \frac{q(t)}{\xi(t)}+E^{\prime}(t) \\
& \leq-\eta E(t) \bar{M}^{\prime}\left(r_{0} \frac{E(t)}{E(0)}\right)+C r_{0} \frac{E(t)}{E(0)} \bar{M}^{\prime}\left(r_{0} \frac{E(t)}{E(0)}\right)+C \omega \frac{q(t)}{\xi(t)}+E^{\prime}(t) .
\end{aligned}
$$

Now, multiplying (78) by $\xi(t)$ keeping in mind that $r_{0} \frac{E(t)}{E(0)}<r$ and $\bar{M}^{\prime}\left(r_{0} \frac{E(t)}{E(0)}\right)=M^{\prime}\left(r_{0} \frac{E(t)}{E(0)}\right)$, we arrive at

$$
\begin{aligned}
\xi(t) K_{3}^{\prime}(t) & \leq-\eta \xi(t) E(t) M^{\prime}\left(r_{0} \frac{E(t)}{E(0)}\right)+C r_{0} \frac{E(t)}{E(0)} \xi(t) M^{\prime}\left(r_{0} \frac{E(t)}{E(0)}\right)+C \omega q(t)+\xi(t) E^{\prime}(t) \\
& \leq-\eta \xi(t) E(t) M^{\prime}\left(r_{0} \frac{E(t)}{E(0)}\right)+C r_{0} \frac{E(t)}{E(0)} \xi(t) M^{\prime}\left(r_{0} \frac{E(t)}{E(0)}\right)-C E^{\prime}(t) .
\end{aligned}
$$

We set $K_{4}(t)=\xi(t) K_{3}(t)+C E(t) \sim E(t)$ since $K_{3} \sim E$. Thus there exist $n_{0}, n_{1}$ positive such that

$$
n_{0} K_{4}(t) \leq E(t) \leq n_{1} K_{4}(t) .
$$

Therefore, estimate (79) yields

$$
K_{4}^{\prime}(t) \leq-\left(\eta E(0)-C r_{0}\right) \xi(t) \frac{E(t)}{E(0)} \xi(t) M^{\prime}\left(r_{0} \frac{E(t)}{E(0)}\right), \forall t \geq t_{0}
$$

We choose $r_{0}<r$ small enough so that $\eta E(0)-C r_{0}>0$ to arrive at

$$
K_{4}^{\prime}(t) \leq-\eta_{2} \xi(t) \frac{E(t)}{E(0)} \xi(t) M^{\prime}\left(r_{0} \frac{E(t)}{E(0)}\right)=-\eta_{2} \xi(t) M_{2}\left(\frac{E(t)}{E(0)}\right), \quad \forall t \geq t_{0}
$$


for some constant $\eta_{2}>0$ and $M_{2}(s)=s M^{\prime}\left(r_{0} s\right)$. We note that $M_{2}^{\prime}(s)=M^{\prime}\left(r_{0} s\right)+r_{0} s M^{\prime \prime}\left(r_{0} s\right)$, hence, the strict convexity of $M$ on $(0, r]$, yields $M_{2}(s)>0, M_{2}^{\prime}(s)>0$ on $(0, r]$. Let $R(t)=n_{0} \frac{K_{4}(t)}{E(0)}$. Using (80) and (81), we obtain

$$
R(t) \sim E(t)
$$

and

$$
R^{\prime}(t)=n_{0} \frac{K_{4}^{\prime}(t)}{E(0)} \leq-\eta_{3} \xi(t) M_{2}(R(t)), \forall t \geq t_{0}
$$

for some $\eta_{3}>0$. Integration of $(83)$ over $\left(t_{0}, t\right)$, gives

$$
\eta_{3} \int_{t_{0}}^{t} \xi(s) d s \leq-\int_{t_{0}}^{t} \frac{R^{\prime}(s)}{M_{2}(R(s))} d s=\frac{1}{r_{0}} \int_{r_{0} R(t)}^{r_{0} R\left(t_{0}\right)} \frac{1}{s M^{\prime}(s)} d s
$$

from which we get

$$
R(t) \leq \frac{1}{r_{0}} M_{1}^{-1}\left(\eta_{3} \int_{t_{0}}^{t} \xi(s) d s\right), \quad \text { where } M_{1}(t)=\int_{t}^{r} \frac{1}{s M^{\prime}(s)} d s .
$$

Using properties of $M$, we easily see that $M_{1}$ is strictly decreasing function on $(0, r]$ and $\lim _{t \rightarrow 0} M_{1}(t)=+\infty$. Therefore, (62) follows from (82) and (85). This completes the proof.

Remark 1. The stability result in Theorem 2 is general and optimal in the sense that it agrees with the decay rate of $g$, see [30], Remark 2.3.

Corollary 1. Suppose $\frac{k}{\rho}=\frac{\delta}{J}$, and $(A 1)-(A 5)$ hold. Assume the function $M$ in $\left(A_{2}\right)$ be defined by $H(s)=s^{p}, p \geq 1$, then there exist $\lambda_{1}, \lambda_{2}, C>0$ such that (20) satisfies

$$
E(t) \leq \begin{cases}\lambda_{2} \exp \left(-\lambda_{1} \int_{0}^{t} \xi(s) d s\right), & \text { for } p=1, \\ \frac{C}{\left(1+\int_{t_{0}}^{t} \xi(s) d s\right)^{\frac{1}{p-1}}}, & \text { for } p>1 .\end{cases}
$$

\section{Examples}

(1). Let $g(t)=v_{1} e^{-v_{2} t}, t \geq 0, v_{1}, v_{2}>0$ and $v_{2}$ is chosen so that $\left(\mathrm{A}_{1}\right)$ holds. Then, $g^{\prime}(t)=-v_{1} v_{2} e^{-v_{2} t}=$ $-v_{2} M(g(t))$ with $M(t)=t$. Thus, from (62), the solution energy (20) satisfies $E(t) \leq C e^{-\lambda t}, \forall t \geq 0$, where $\lambda=v_{2} \lambda_{1}$.

(2). Let $g(t)=u e^{-(1+t)^{v}}, t \geq 0, u>0,0<v<1$ are constants and $u$ is chosen such that $\left(\mathrm{A}_{1}\right)$ holds. Then, $g^{\prime}(t)=-u v(1+t)^{v-1} e^{-(1+t)^{v}}=-\xi(t) M(g(t))$, where $\xi(t)=v(1+t)^{v-1}$ and $M(t)=t$. Thus, we get from (62) that $E(t) \leq \lambda_{2} e^{-\lambda_{1}(1+t)^{v}}, \forall t \geq 0$.

(3). Let $g(t)=\frac{u}{(1+t)^{v}}, t \geq 0, u>0, v>1$ are constants and $u$ is chosen such that $\left(\mathrm{A}_{1}\right)$ holds. We have $g^{\prime}(t)=\frac{-u v}{(1+t)^{v+1}}=-\xi\left(\frac{u}{(1+t)^{v}}\right)^{\frac{v+1}{v}}=-\xi^{p}(t)=-\xi M(g(t))$, where $M(t)=t^{p}, \quad p=\frac{v+1}{v}$ satisfying $1<p<2$ and $\xi=\frac{v}{u^{\frac{1}{v}}}>0$. Hence, we deduce from (86) that $E(t) \leq \frac{C}{(1+t)^{v}}, \forall t \geq 0$.

Acknowledgments: The author appreciate the continuous support of University of Hafr Al Batin.

Conflicts of Interest: "The author declares no conflict of interest."

\section{References}

[1] Chiriţă, S., Ciarletta, M., \& D'Apice, C. (2013). On the theory of thermoelasticity with microtemperatures. Journal of Mathematical Analysis and Applications, 397(1), 349-361.

[2] Cowin, S. C. (1985). The viscoelastic behavior of linear elastic materials with voids. Journal of Elasticity, 15(2), 185-191.

[3] Cowin, S. C., \& Nunziato, J. W. (1983). Linear elastic materials with voids. Journal of Elasticity, 13(2), 125-147.

[4] Goodman, M. A., \& Cowin, S. C. (1972). A continuum theory for granular materials. Archive for Rational Mechanics and Analysis, 44(4), 249-266.

[5] Ieşan, D. (1986). A theory of thermoelastic materials with voids. Acta Mechanica, 60(1), 67-89.

[6] Ieşan, D. (2004). Thermoelastic Models of Continua (Vol. 118). Springer Science \& Business Media. 
[7] Nunziato, J. W., \& Cowin, S. C. (1979). A nonlinear theory of elastic materials with voids. Archive for Rational Mechanics and Analysis, 72(2), 175-201.

[8] Quintanilla, D. I. R. (2000). On a theory of thermoelasticity with microtemperatures. Journal of Thermal Stresses, 23(3), 199-215.

[9] Nicaise, S., \& Pignotti, C. (2006). Stability and instability results of the wave equation with a delay term in the boundary or internal feedbacks. SIAM Journal on Control and Optimization, 45(5), 1561-1585.

[10] Kafini, M., Messaoudi, S. A., \& Nicaise, S. (2016). A blow-up result in a nonlinear abstract evolution system with delay. Nonlinear Differential Equations and Applications NoDEA, 23(2), 13.

[11] Liu, W. (2013). General decay of the solution for a viscoelastic wave equation with a time-varying delay term in the internal feedback. Journal of Mathematical Physics, 54(4), 043504.

[12] Liu, W. (2013). General decay rate estimate for the energy of a weak viscoelastic equation with an internal time-varying delay term. Taiwanese Journal of Mathematics, 17(6), 2101-2115.

[13] Nicaise, S., \& Pignotti, C. (2011). Interior feedback stabilization of wave equations with time dependent delay. Electronic Journal of Differential Equations, 2011(41), 1-20.

[14] Messaoudi, S. A., Fareh, A., \& Doudi, N. (2016). Well posedness and exponential stability in a wave equation with a strong damping and a strong delay. Journal of Mathematical Physics, 57(11), 111501.

[15] Mukiawa, S. E. (2020). Decay result for a delay viscoelastic plate equation. Bulletin of the Brazilian Mathematical Society, New Series, 51(2), 333-356.

[16] Quintanilla, R. (2003). Slow decay for one-dimensional porous dissipation elasticity. Applied Mathematics Letters, 16(4), 487-491.

[17] Casas, P. S., \& Quintanilla, R. (2005). Exponential decay in one-dimensional porous-thermo-elasticity. Mechanics Research Communications, 32(6), 652-658.

[18] Soufyane, A., Afilal, M., Aouam, T., \& Chacha, M. (2010). General decay of solutions of a linear one-dimensional porous-thermoelasticity system with a boundary control of memory type. Nonlinear Analysis: Theory, Methods $\mathcal{E}$ Applications, 72(11), 3903-3910.

[19] Apalara, T. A. (2019). General decay of solutions in one-dimensional porous-elastic system with memory. Journal of Mathematical Analysis and Applications, 469(2), 457-471.

[20] Feng, B., \& Apalara, T. A. (2019). Optimal decay for a porous elasticity system with memory. Journal of Mathematical Analysis and Applications, 470(2), 1108-1128.

[21] Khochemane, H. E., Zitouni, S., \& Bouzettouta, L. (2020). Stability result for a nonlinear damping porous-elastic system with delay term. Nonlinear studies, 27(2), 487-503.

[22] Borges Filho, E., \& Santos, M. L. (2020). On porous-elastic system with a time-varying delay term in the internal feedbacks. ZAMM-Journal of Applied Mathematics and Mechanics Zeitschrift für Angewandte Mathematik und Mechanik, 100(8), e201800247.

[23] Dos Santos, M. J., Feng, B., Júnior, D. S. A., \& Santos, M. L. (2021). Global and exponential attractors for a nonlinear porous elastic system with delay term. Discrete $\mathcal{E}$ Continuous Dynamical Systems-B, 26(5), 2805-2828.

[24] Feng, B. (2019). On the decay rates for a one-dimensional porous elasticity system with past history. Communications on Pure E Applied Analysis, 18(6), 2905.

[25] Feng, B., \& Yin, M. (2019). Decay of solutions for a one-dimensional porous elasticity system with memory: the case of non-equal wave speeds. Mathematics and Mechanics of Solids, 24(8), 2361-2373.

[26] Kafini, M., Messaoudi, S. A., \& Mustafa, M. I. (2014). Energy decay rates for a Timoshenko-type system of thermoelasticity of type III with constant delay. Applicable Analysis, 93(6), 1201-1216.

[27] Santos, M. L., \& Júnior, D. A. (2016). On porous-elastic system with localized damping. Zeitschrift für angewandte Mathematik und Physik, 67(3), 63.

[28] Feng, B. (2017). General decay for a viscoelastic wave equation with strong time-dependent delay. Boundary Value Problems, 2017, Article No. 57.

[29] Arnol'd, V. I. (2013). Mathematical Methods of Classical Mechanics (Vol. 60). Springer Science \& Business Media.

[30] Mustafa, M. I. (2018). General decay result for nonlinear viscoelastic equations. Journal of Mathematical Analysis and Applications, 457(1), 134-152. 